\title{
A 100MHz PRF IR-UWB CMOS Transceiver with Pulse Shaping Capabilities and Peak Voltage Detector
}

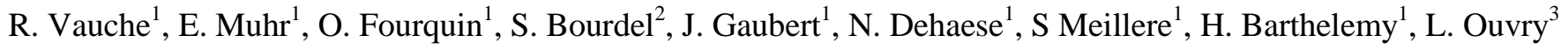

\author{
${ }^{1}$ Aix Marseille Univ, Univ Toulon, CNRS, IM2NP, Marseille, France \\ ${ }^{2}$ Université Grenoble-Alpes, CNRS, IMEP-LAHC UMR 5130, F-38000 Grenoble, France \\ ${ }^{3}$ Université Grenoble Alpes, F-38000 Grenoble, France, CEA, LETI, MINATEC Campus, F-38054 Grenoble, France
}

\begin{abstract}
This work presents a high rate IR-UWB transceiver chipset implemented in a $130 \mathrm{~nm}$ CMOS technology for WBAN and biomedical applications in the 3.1GHz-4.9GHz band. The transmitter is based on a pulse synthesizer and an analytical upconverted Gaussian pulse is used to predict its settings. Its measured peak to peak output voltage is equal to $0.9 \mathrm{Vpp}$ on a $100 \Omega$ load for a central frequency of $4 \mathrm{GHz}$, and a supply voltage of $1.2 \mathrm{~V}$, which gives an emitted energy per pulse of $0.64 \mathrm{pJ}$. The receiver is a non-coherent architecture based on a LNA followed by a peak voltage detector. A BER of $10^{-3}$ is measured for a 3.1GHz-4.9GHz input peak-to-peak pulse amplitude of $1.1 \mathrm{mV}$

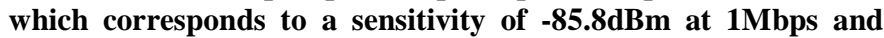
gives a communication range estimated to $1.9 \mathrm{~m}$.
\end{abstract}

Index Terms - IR-UWB, Ultra-Wideband CMOS transceiver, WBAN, non-coherent receiver, Pulse shaping Capabilities

\section{INTRODUCTION}

Ultra-WideBand Impulse Radio (IR-UWB) is a well-known technique based on the transmission of short duration pulses. Such approach is well suited for low range, medium rate and low power applications such as Body Area Network (BAN), Wireless Sensor Network (WSN) or ranging [1][2]. Since the Federal Communications Commission (FCC) has approved UWB communications in the $3.1-10.6 \mathrm{GHz}$ bandwidth, many successful works have been reported showing the ease of making energy efficient systems with IR-UWB.

Recently, the research field has moved towards new challenges such as system reliability, integration and cost issues or high energy efficiency. In this context, a few meter range $100 \mathrm{Mbps}$ transceiver designed for BAN applications is presented. Here, the approach is to alleviate as much as possible the system constraints while allowing high tuning capabilities to ensure a successful design.

High energy efficiency is intrinsically based on the gated nature of the IR-UWB signal. To fully exploit this property, duty-cycled systems can be used. A first approach consists in duty-cycling the system at the bit rate [1] which improves the system immunity to narrow band interferers and reduces power consumption. For low bit rates (less or equal to $1 \mathrm{Mbps}$ ), an on-time less than ten percent of the bit time can be reached with fast turn-on devices and pulse coupled oscillator based architectures for synchronization [3]. A lower on-time (close to 30ns) has been reached with a $100 \mathrm{MHz}$ All Digital Clock and Data Recovery (AD-CDR) having 10ns jitter [4]. However, when a medium rate (e.g., 15.6Mbps) is considered, the Pulse Repetition Period (PRP) is around a few tens of nanoseconds (e.g., 66.7ns) and the precision of the dutycycling clock must be high enough (a few nanoseconds) in order to reduce efficiently the power consumption. In this case, the clock frequency of the AD-CDR must be increased up to $1 \mathrm{GHz}$ which impacts the system power consumption [4]. Solutions based on synchronized modulation schemes [5] have also been proposed. However, they require the transmission of additional pulses for synchronization which reduces the energy efficiency and the payload bit rate of the system.

For medium rate, a solution to reduce power consumption is to achieve a duty-cycling at burst rate. As presented in [6], this approach can reach the same energy efficiency as in a bit rate duty-cycled system, while the duty-cycling clock precision is moved towards a few tens of microseconds which releases constraints on the CDR jitter characteristics. In this case, the required idle time $T_{I D L E}$ to maintain constant the Power Spectral Density (PSD) is equal to $T_{\text {SWEEP }}\left(1-P R F_{M E A N} /\right.$ $P R F_{\text {BURST }}$ ) where $T_{\text {SWEEP }}$ is the sweep time imposed by FCC to measure the PSD (1ms), PRF BURST the Pulse Repetition Frequency (PRF) during the burst, and $P R F_{M E A N}$ the mean PRF averaged on $T_{S W E E P}$. By considering $P R F_{M E A N}$ (resp. $P R F_{\text {BURST }}$ ) is equal to $15.6 \mathrm{MHz}$ (resp. $100 \mathrm{MHz}$ ), the ideal ontime $T_{O N}$ is $156 \mu \mathrm{s}$, the ideal idle time $T_{I D L E}$ is $844 \mu \mathrm{s}$, and the number of pulses per burst is $15.6 \mathrm{k}$. In the context of a dutycycled at burst level, the synchronization scheme is no more an issue and is not addressed in this paper.

However, a high rate capability (around $100 \mathrm{MHz}$ ) is decisive to minimize the ratio $P R F_{\text {MEAN }} / P R F_{B U R S T}$ and to maximize the power reduction. Such approach also requires a high data rate CDR which can be duty cycled at the burst rate like the receiver. Moreover, the acquisition time of an ADCDR will not impact the energy efficiency because the number of pulses required for the acquisition (32 in [4]) is negligible regarding the number of pulses in the burst.

Regarding the transmitter, controlling the radiated spectrum is necessary to comply with standard masks or to reject spectrum side-lobes. Passive solutions based on external filters or integrated filters can be used [7] to shape the pulse but they dramatically impact the size and the cost. Solutions based on active devices [8][9] suite better the integration issues but need high tuning capabilities to compensate Process Voltage and Temperature (PVT) variations. In this context, several solutions have been proposed to control the shape of the 
pulses [10][11][12]. Among these solutions, digital synthesis [12] is a promising approach because it operates without inductor and allows a fine tuning of the pulse shape to be obtained.

On the receiver side, Non-Coherent (NC) detection is often used to reduce complexity and save power in IR-UWB receivers. Among the different architectures, energy detectors based on super-regenerative or self-mixing coupled to integrators achieve good sensitivity performances [13][14]. However, the need for synchronization schemes operating at symbol rate increases the integration issues and reduces the maximum achievable bit rate. Another way of designing NC receivers is to use Peak Voltage Detectors (PVD). On the one hand, PVDs suffer from a lower theoretical sensitivity since the signal is not collected all along the symbol duration time as it is in energy detectors. However, using PVDs can be justified in case of a short-range transceiver. On the other hand, PVDs are intrinsically asynchronous and are well suited for a burst duty-cycled receiver.

In this paper, a high rate $3.1 \mathrm{GHz}-4.9 \mathrm{GHz}$ IR-UWB transceiver for WBAN applications is presented. It uses a pulse synthesizer with pulse shaping capabilities on the emitter side and a PVD at the receiver side. Section II gives a system overview and analyses the theoretical performance in terms of Bit Error Rate (BER). Since the transmitter architecture is based on pulse synthesis, an analytical pulse model is proposed to predict the required pulse shape. Next, this shape is used to estimate BER performance of the peak voltage detector implemented in the receiver. Section III and section IV present respectively the transmitter and the receiver parts of the transceiver. In Section V, measurement results obtained with the proposed transceiver are presented and compared with previous published works.

\section{SYSTEM OVERVIEW}

In this section, the proposed IR-UWB transmitter, which is based on pulse synthesis technique, is presented and an analytical model of Gaussian up-converted pulse is proposed to predict the waveform to generate according to the targeted bandwidth. Next, after a presentation of the proposed NC IRUWB receiver based on a peak voltage detector, its BER performance is estimated with the help of the presented pulse model.

\section{A. Proposed IR-UWB Transmitter}

To communicate in the allocated FCC band, an IR-UWB transmitter requires a pulse generator to transmit data. Lots of approaches have been developed to generate UWB signals but they have generally few capabilities regarding spectrum shapes programmability.

Solutions based on the modulation of a carrier by an analog signal, such as a triangular waveform or a raised cosine, have been proposed [15][16] in order to shape the envelop of the emitted pulse, to better fit standard masks, and to reduce side lobes. This approach is efficient in terms of side lobe rejection but lacks of reconfiguration and tuning capabilities. All digital architectures, which are more flexible than analog ones, have been proposed. They allow the pulse shape to be tuned in case of PVT variations [10][17]. All-digital pulse generators are generally based on Digitally Controlled Oscillator (DCO) [11], Digital Delay Line (DDL) [10], or both [17]. Compared to a DCO based generator, a DDL based pulse generator can be easily duty-cycled to reduce the power consumption since it requires shorter start-up time. Moreover, it is possible to adjust each sub-lob of the pulse to accurately tune the envelop and control the side-lobe level.

As shown in Fig. 1, an UWB pulse $p(t)$ can be seen as a linear combination of $\mathrm{N}$ baseband pulses $\left(g_{n}(t)\right)$ as follows:

$$
p(t)=\sum_{n=1}^{N} A_{n} \cdot g_{n}\left(t-\sum_{p=1}^{n-1} \tau_{p}\right)
$$

where $A_{n}$ and $\tau_{n}$ are respectively magnitude and width of the $n^{\text {th }}$ baseband pulse. To synthesize $p(t)$, two circuits can be associated, a $N$ baseband pulses generator and a $N$ baseband pulses combiner, as shown in Fig. 1. The baseband pulses generator allows each baseband pulse (from $n=1$ to $N$ ) to be generated according to the targeted width ( $\tau_{n}$ from $n=1$ to $N$ ). Next, baseband pulses are combined by the baseband pulses combiner according to the targeted magnitude $\left(A_{n}\right.$ from $n=1$ to $N)$. The proposed IR-UWB transmitter is based on this approach.

To optimize the bit rate and/or the communication range with a $\mathrm{NC}$ receiver while the maximum mean power given by FCC is respected, it is proposed here to use a Random Alternate On-Off Keying modulation (RA-OOK) [6]. This modulation does not have discrete spectrum in its PSD but is similar to an OOK modulation from the receiver point of view. Indeed, to send a binary ' 0 ', no pulse is emitted. However, to send a binary ' 1 ', a positive pulse or a negative pulse is randomly sent.

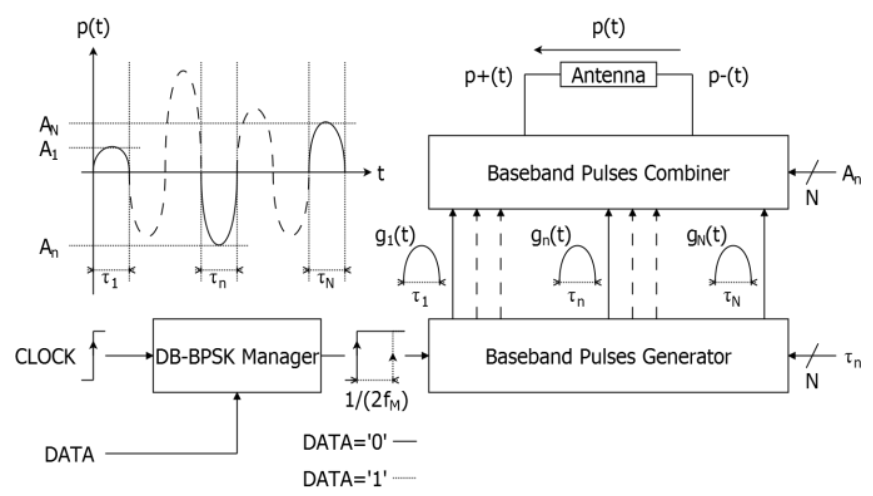

Fig. 1. Proposed IR-UWB transmitter based on pulse synthesizing technique. 
To generate these positive and negative pulses, a DelayBased Binary Phase Shift Keying (DB-BPSK) modulator can be used [7][18]. It is based on a delay cell which will softly delay or not the CLOCK triggered signal according to the DATA logical state indicated in Fig. 1. This allows positive and negative pulses of a BPSK modulation to be approximated and also allows the discrete spectrum of the emitted PSD to be suppressed which reduces the mean emitted power. To obtain the best results, the CLOCK delay $\left(\tau_{D B-B P S K}\right)$ must be equal to $1 /\left(2 \cdot f_{M}\right)$ with $f_{M}$, the frequency where the emitted power is maximum [18]. In this case, the mean emitted power can be increased until the authorized limit by increasing the bit rate and/or the energy of each pulse [7].

This DB-BPSK modulator has been implemented in the proposed IR-UWB transmitter and it is also used to obtain a Random Alternate On-Off Keying modulation [6] by using the following rules. To send a binary ' 0 ', no rising edge is provided on CLOCK and no pulse is emitted. To send a binary ' 1 ', a rising edge is provided on CLOCK and a positive or negative pulse is emitted according to the state of DATA.

\section{B. UWB pulse waveform estimation}

The use of pulse synthesizers as IR-UWB transmitters implies the definition of an UWB pulse which can match with the targeted bandwidth, in order to get an ad hoc configuration for $A_{n}$ and $\tau_{n}$ control inputs. To define this pulse, it is possible to compute the inverse Fourier transform of the targeted frequency mask, or to analyze the impulse response of a filter which has a compliant frequency response. The fifth [19][20] and the seventh [21][22] Gaussian derivatives can also be used especially to match with FCC mask. However, these methods lead to a high number $\mathrm{N}$ of couples $\left(A_{n}, \tau_{n}\right)$ to synthesize when a $3.1 \mathrm{GHz}-4.9 \mathrm{GHz}$ bandwidth is targeted. To address this bandwidth with a few couples $\left(A_{n}, \tau_{n}\right)$, triangular or Gaussian up-converted pulses have to be preferred. However, the Gaussian up-converted pulse has a better spectral efficiency $\eta_{S E}$ than the triangular one. This spectral efficiency $\eta_{S E}$ can be defined as follows:

$$
\eta_{S E}=\frac{\frac{1}{Z_{L}} \int_{\mathbb{R}} p^{2}(t) \cdot d t}{E_{R E F}}=\frac{B W_{-X d B}}{A^{2}} \int_{\mathbb{R}} p^{2}(t) \cdot d t
$$

where $Z_{L}$ is the impedance load of the antenna connected to the pulse generator output, and $E_{R E F}$ is the energy of a reference pulse which is here an up-converted cardinal sine having the same magnitude $(A)$ of the emitted pulse and occupying the same bandwidth $\left(B W_{-X d B}\right)$. In this case, it appears that the Gaussian up-converted pulse has an efficiency of $86 \%$ instead of $56 \%$ for the triangular one. Thus, to keep $N$ low and a high emitted energy distribution, an odd Gaussian up-converted pulse is proposed here. It only requires $N / 2$ values of $A_{n}$ in addition to one $\tau_{n}$ value. Moreover, it can be easily tuned to match with different spectrum masks since the Fourier transform of a Gaussian function is another analytically computable Gaussian function.

The proposed odd Gaussian up-converted pulse can be written in time domain as follows:
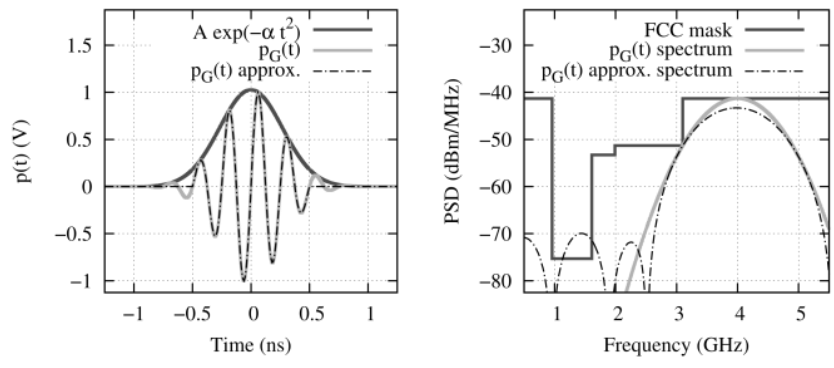

Fig. 2. Example of transient and frequency responses of the proposed Gaussian up-converted pulses with $A_{P P}=2 \mathrm{~V}, f_{M}=4 \mathrm{GHz}$, and $B W_{-10 \mathrm{~dB}}=1.8 \mathrm{GHz}$.

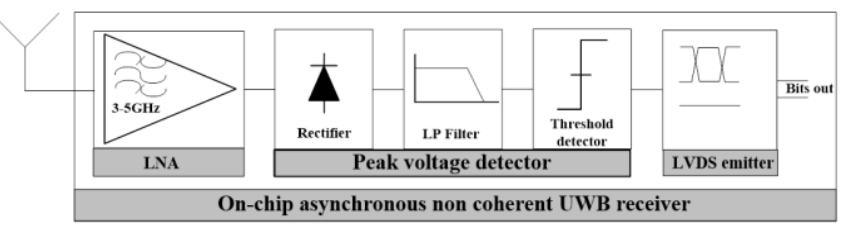

Fig. 3. Proposed IR-UWB NC receiver based on peak voltage detection.

$$
p_{G}(t)=A \exp \left(-\alpha t^{2}\right) \sin \left(2 \pi f_{M} t\right)
$$

where $A$ is the maximum of the Gaussian envelope and $\alpha$, a parameter which sets the pulse bandwidth. In this case, the width $\tau_{n}$ of every baseband pulses is also equal to the optimum value of $\tau_{D B-B P S K}$ which is $1 /\left(2 \cdot f_{M}\right)$. The maximum of the Gaussian envelope $A$ can be related to the peak to peak magnitude $A_{P P}$ of the pulse as follows:

$$
A=\frac{A_{P P}}{2} \exp \left[\frac{\alpha}{\left(4 f_{M}\right)^{2}}\right] .
$$

The magnitude of the single side Fourier transform of $p_{G}(t)$ can be written as follows:

$$
\left|\hat{p}_{G}(f)\right|=A \sqrt{\frac{\pi}{2 \alpha}} \exp \left(-\frac{\left[2 \pi\left(f-f_{M}\right)\right]^{2}}{4 \alpha}\right)
$$

and allows the $\alpha$ parameter to be computed as a function of the targeted bandwidth $B W_{-X \mathrm{~dB}}$ defined at $-X \mathrm{~dB}$ as follows:

$$
\alpha=\frac{\pi^{2} B W_{-X \mathrm{~dB}}^{2}}{2 \ln \left(10^{X \mathrm{~dB} / 10}\right)} .
$$

Moreover, the energy emitted per pulse $E_{P-G}$ can be computed from (4) and is equal to:

$$
E_{P-G}=\frac{A^{2}}{2 Z_{L}} \sqrt{\frac{\pi}{2 \alpha}}\left[1+\exp \left(-\frac{\left[4 \pi f_{M}\right]^{2}}{8 \alpha}\right)\right]
$$

This result can be approximated with an error of 5\% with the following expression:

$$
E_{P-G} \simeq \frac{A^{2}}{2 Z_{L} B W_{-X \mathrm{~dB}}} \sqrt{\frac{\ln \left(10^{X \mathrm{~dB} / 10}\right)}{\pi}}
$$


and enables energy estimation for other works comparison with only few parameters [6].

An example of this Gaussian up-converted pulse is shown in Fig. 2 for the targeted 3-5GHz bandwidth. However, as the Gaussian envelope defined here by $A \cdot \exp \left(-\alpha \cdot t^{2}\right)$ is never equal to zero and that an infinite duration pulse cannot be synthesized, it is necessary to approximate $p_{G}(t)$. In Fig. 2, $p_{G}(t)$ is approximated by the height higher monocycle of the Gaussian pulse. This approximation shows a loss of $2 \mathrm{~dB}$ in frequency domain and an unwanted side lobe around $1.5 \mathrm{GHz}$. The both can be corrected thanks to pulse synthesis by using a fine tuning of $A_{n}$ values.

\section{Proposed Non-Coherent Receiver}

Usually Non-Coherent UWB receivers use energy detection for pulse detection. In these architectures an image of the pulse energy is obtained by squaring and integrating the incoming signal during the symbol duration time. The signal amplitude at the squarer output, which varies as the square of the amplitude of the input signal, quickly becomes very small and unusable when the receiver moves away from the transmitter. Consequently, in order to obtain signals which exceed the noise floor of the stages downstream of the squarer, a large amount of amplification must be provided by the Low Noise Amplifier (LNA), whereas achieving high amplification gain with large bandwidth is difficult in low cost CMOS technologies. Although it is less efficient from a theoretical point of view, a NC receiver based on peak voltage detection could provide an interesting alternative which relaxes design constraints regarding the LNA gain, while being compatible with the requirements of many short range applications.

The proposed NC receiver architecture which uses peak voltage detection is described in Fig. 3. It is composed of a band pass type LNA that drives an asynchronous peak detector based on envelope detection. The peak detector provides a short duration (1.5ns) baseband pulse for each incoming UWB pulse. The single ended numeric signal is then converted into a Low Voltage Differential Signaling (LVDS) signal in order to provide standardized signals to the board interface.

\section{Performance analysis of peak voltage detection}

In this section, an estimation of the BER which can be achieved with peak voltage detection is presented by considering an OOK coding, an AWGN channel, and no multipath propagation.

As shown in [23][24], the dimensionality of the space of finite energy signals is about $2 \cdot B \cdot T+1$ with a bandwidth $B$ and a time spread $T$. From its equivalent formula in the discrete domain, it is possible to perform the BER calculation. In this work, it is then assumed that, during the symbol duration time $T_{S}$, the continuous time domain signal at the receiver input can be replaced by $P$ discrete samples of the received signal obtained at the Nyquist rate:

$$
P=2 \cdot f_{\text {MAX }} \cdot T_{S}+1 \text {. }
$$

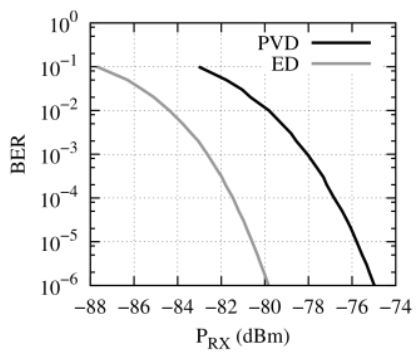

(a)

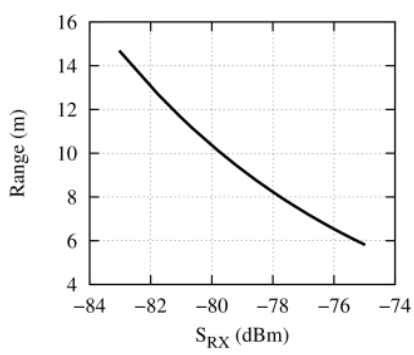

(b)
Fig. 4. Bit error rate versus the received power for OOK modulated $3.1 \mathrm{GHz}-$ $4.9 \mathrm{GHz}$ pulses at a rate of $100 \mathrm{Mbps}$ and with $\mathrm{NF}_{\mathrm{LNA}}=3 \mathrm{~dB}$ for a Peak Voltage Detector (PVD) and an Energy Detector (ED) (a). Theoretical range with PVD as a function of the receiver sensitivity when a $0.52 \mathrm{pJ}(0.45 \mathrm{~V}$ peak magnitude) pulse is emitted on a $2 \times 50 \Omega$ differential antenna (b).

Given that, for the $3.1 \mathrm{GHz}-4.9 \mathrm{GHz}$ bandwidth UWB signal considered here, the noise bandwidth $(1.8 \mathrm{GHz})$ is close to the maximum spectrum frequency $\mathrm{f}_{\mathrm{MAX}}(4.9 \mathrm{GHz})$, we also assume that the noise samples are independent. The discrete samples of the received signal are the sum of the samples of the unnoisy signal and of the noise. Assuming an AWGN channel and the independence of the noise samples, the probability density function of the noise voltage for each sample is given by:

$$
p_{N}(v)=\frac{1}{\sqrt{2 \pi \sigma_{N}^{2}}} \exp \left(\frac{-v^{2}}{2 \sigma_{N}^{2}}\right) .
$$

Assuming that the noise bandwidth is the same that the bandwidth $(B W)$ of the signal, the noise variance $\sigma_{N}$ at the LNA output is given by:

$$
10 \log \left(\frac{\sigma_{N}^{2}}{R_{L N A}}\right)=10 \log (K \cdot T \cdot B W)+G_{L N A}+N F_{L N A}
$$

where $K$ is the Boltzmann constant, $T$ the absolute temperature, $R_{L N A}, G_{L N A}$ and $N F_{L N A}$ the input resistor, the gain, and the noise figure of the LNA.

For an OOK coding, the error probability is given by:

$$
\operatorname{Pr}_{E}=0.5 \cdot \operatorname{Pr}_{F A}+0.5 \cdot \operatorname{Pr}_{N D}
$$

where $\operatorname{Pr}_{\mathrm{FA}}$ is the probability of a false alarm and $\operatorname{Pr}_{\mathrm{ND}}$ is the probability of a non-detection.

A false alarm occurs when the detector output switches to the high level while no pulse has been sent during the symbol duration time. So a false alarm occurs when the noise level crosses the value of the decision threshold $S_{D}$ for at least one of the noisy samples during the symbol duration time. So, the probability of a false alarm is given by:

$$
\operatorname{Pr}_{F A}=1-\left[1-\frac{1}{\sqrt{2 \pi \sigma_{N}^{2}}} \int_{S_{D}}^{+\infty} \exp \left(\frac{-v^{2}}{2 \sigma_{N}^{2}}\right) d v\right]^{P}
$$


where $P$ is the number of samples during the symbol duration time $T_{S}$ given by (9).

A non-detection occurs when a pulse is sent and the magnitude of the noisy signal remains lower than the decision threshold during the symbol duration time. This corresponds for the equivalent discrete signal when no noisy signal sample crosses the value of the decision threshold $S_{D}$ during the symbol duration time. So the probability of a non-detection is given by:

$$
\operatorname{Pr}_{N D}=\prod_{i=0}^{P}\left[\frac{1}{\sqrt{2 \pi \sigma_{N}^{2}}} \int_{-\infty}^{S_{D}-V_{S}\left(t_{i}\right)} \exp \left(\frac{-v^{2}}{2 \sigma_{N}^{2}}\right) d v\right]
$$

where $V_{S}\left(t_{i}\right)$ is the magnitude of the discrete samples of the unnoisy signal at the peak voltage detector input. The shape of $V_{S}\left(t_{i}\right)$ depends on the signal bandwidth and its magnitude depends on the received power and the LNA gain. In our case $V_{S}\left(t_{i}\right)$ is computed from (3) knowing the emitted power at the emitter side, the communication range, and the LNA gain.

For a given received power, a given data rate and a given decision threshold, the BER value is obtained with the equations (12), (13) and (14). For a given BER, there is an optimal decision threshold $S_{D}$ that minimizes the received power and balances $\operatorname{Pr}_{F A}$ and $\operatorname{Pr}_{N D}$. So, by using equations (12), (13), (14) and an iterative algorithm, the receiver sensitivity can be obtained for a given BER. Finally, the use of the Friis formula with this minimum received input power gives the achievable range for a given emitted power. By using the UWB pulse defined by (3) for a data rate of $100 \mathrm{Mbps}$ with an OOK encoding and a $1.8 \mathrm{GHz}-10 \mathrm{~dB}$ bandwidth centered at $4 \mathrm{GHz}$, the results plotted in Fig. 4 are obtained. The same analysis has been done for an ideal Energy Detector (ED) in the appendix. Results are also shown in Fig. 4 for an integration time equal to the pulse width (2ns) when a 100Mbps OOK is considered. Results are also shown in Fig. 4 and even if the peak voltage detection shows a lower theoretical sensitivity than energy detection, it is sufficient for many short range applications. Indeed, it is possible to observe in Fig. 4 that a theoretical range of $8.28 \mathrm{~m}$ is achievable with a peak voltage detection for a BER value of $10^{-3}$ when a power of $-78 \mathrm{dBm}$ is received. This range is obtained when a $0.9 \mathrm{Vpp}$ pulse on a $2 \times 50 \Omega$ differential antenna is considered on the emitter side, which is achievable in CMOS technologies [7].

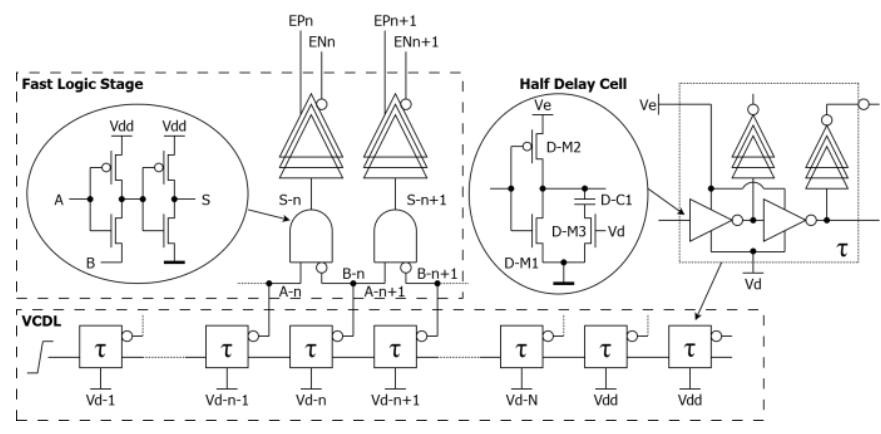

Fig. 5. Design of the proposed baseband pulses generator.

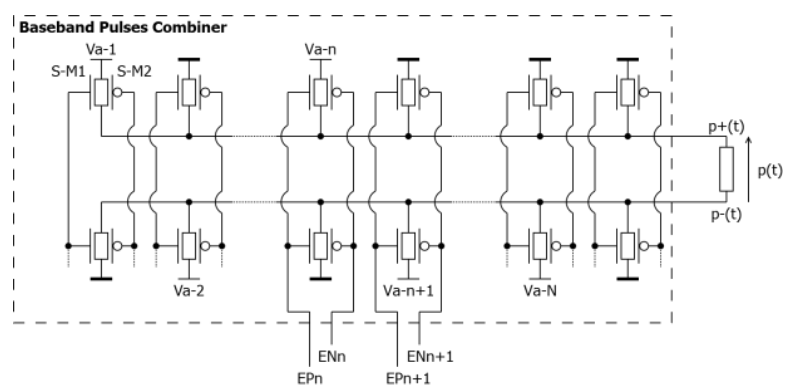

Fig. 6. Design of the proposed baseband pulses combiner.

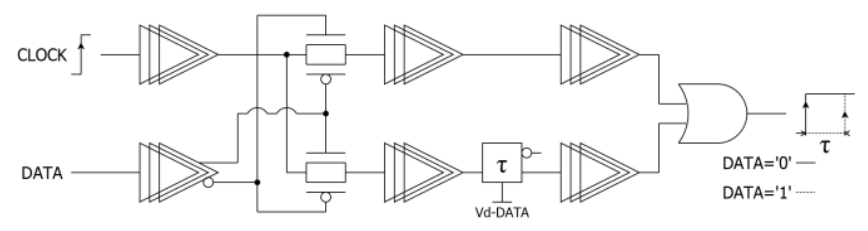

Fig. 7. Design of the proposed DB-BPSK manager.

\section{TRANSMITTER DESIGN}

The presented pulse synthesizer shown in Fig. 1 has been designed in order to synthesize UWB pulses for bandwidths upper than $1.5 \mathrm{GHz}$ centered on a frequency between $3 \mathrm{GHz}$ and $5 \mathrm{GHz}$. To satisfy the targeted bandwidth, the designed pulse synthesizer is able to generate UWB pulses composed of 1 to 8 baseband pulses. All the baseband pulse widths $\tau_{n}$ are controlled by the same biasing voltage except the first and the last in order to compensate for their slower responses due to their positions. Finally, a DB-BPSK pulse manager is integrated in order to implement RA-OOK modulation which allows bit rate and/or pulse energy to be increased while legal maximum powers are respected. The design of each part shown in Fig. 1 is now presented.

\section{A. Baseband Pulses Generator Design}

The baseband pulses generator is composed of two parts, a Voltage Controlled Delay Line (VCDL), and a fast logic stage as shown in Fig. 5.

The VCDL is here a delay line where a rising edge is propagated through tunable delay cells. Each delay cell is built of two buffered stages based on a CMOS inverter loaded by a capacitor (D-C1) in series with a NMOS analog switch (DM3) which gives a large delay tuning range. The width of each baseband pulse $\left(\tau_{n}\right)$ depends on the propagation delay time of the $\mathrm{n}^{\text {th }}$ stage of the VCDL which is set by D-M3, and D-C1 sizes and $\mathrm{V}_{\mathrm{d}-\mathrm{n}}$ value. In this implementation, all $V_{d-n}$ are connected to the same control voltage since the proposed odd up-converted Gaussian pulse requires similar $\tau_{n}$, except for the first $\left(V_{d-1}\right)$ and the last $\left(V_{d-N}\right)$ delay cells which are slower.

Next, the fast logic stage combines rising edges at the output of the $\mathrm{n}^{\text {th }}$ cell $(A)$ and at the output of the $\mathrm{n}+1^{\text {th }}$ cell $(B)$ using a fast logic gate to achieve the $\mathrm{n}^{\text {th }}$ baseband pulse $\left(g_{n}(t)\right)$. To increase the switching speed and also the maximum pulse central frequency, the fast logic stage has been modified from the architecture presented in [25]. Then, the number of nodes has been reduced in order to limit the parasitic capacitances due to interconnections. However, this fast logic stage is able 
to generate the complementary baseband pulses required by the baseband pulses combiner (EPn and ENn).

\section{B. Baseband Pulses Combiner Design}

To generate UWB pulses with 1 to 8 baseband pulses, the baseband pulses combiner is based on $8 \mathrm{H}$-bridge paths going through the load as shown in Fig. 6. Each H-bridge path is composed of two transmission gates (S-M1 and S-M2) and is driven by the balanced baseband pulses on both sides of the load. The magnitude of the recombined baseband pulses $\left(A_{n}\right)$ is controlled by applying different control voltages $\left(V_{a-n}\right)$ at the top of each H-bridge path. An advantage of this structure is that the obtained $A_{n}$ is quasi-proportional to $V_{a-n}$ and also can be easily controlled. To alternate the sign of the baseband pulses, $V_{a-n}$ are alternatively applied to the top and to the bottom of the paths. The baseband pulses combiner has been designed in order to be able to drive a $100 \Omega$ differential antenna with a peak-to-peak magnitude equals to the supply voltage in post-layout simulations (S-M1 and S-M2 widths are respectively equal to $200 \mu \mathrm{m}$ and $480 \mu \mathrm{m}$ ).

\section{DB-BPSK Manager Design}

As shown in Fig. 7, the DB-BPSK manager consists of two paths, one where CLOCK is delayed, and another one where CLOCK is not delayed. It uses standard CMOS logic gates as buffers, transmission gates, and an OR gate. However, it is equally composed of one delay cell which allows CLOCK rising edge to be delayed when DATA is equal to ' 1 '. As the delay cell is the same as the one used in the VCDL, and that all baseband pulses of the synthesized pulse have the same time duration $\left(\tau_{n}\right), V_{d-D A T A}$ has to be connected to $V_{d-n}$ to obtain $\tau_{D B-B P S K}$ equal to $1 /\left(2 \cdot f_{M}\right)$. In this configuration, the DB-BPSK modulation is close as much as possible to the BPSK one in terms of spectrum. To ensure the realization of this

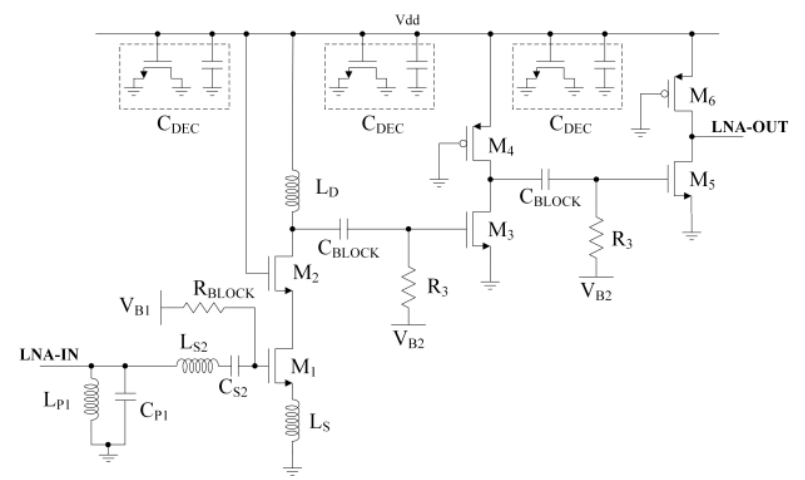

Fig. 8. Schematic of the low noise amplifier circuit.

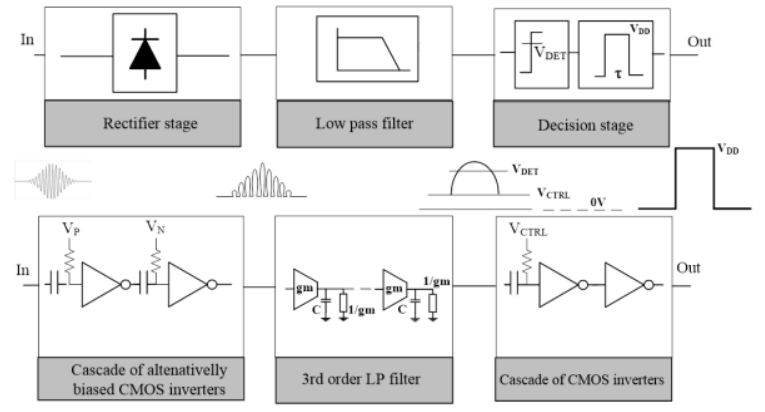

Fig. 9. Peak detector principle and architecture. configuration, buffers are placed on the both sides of the delay cell, and the same buffers are equally placed on the no delayed path.

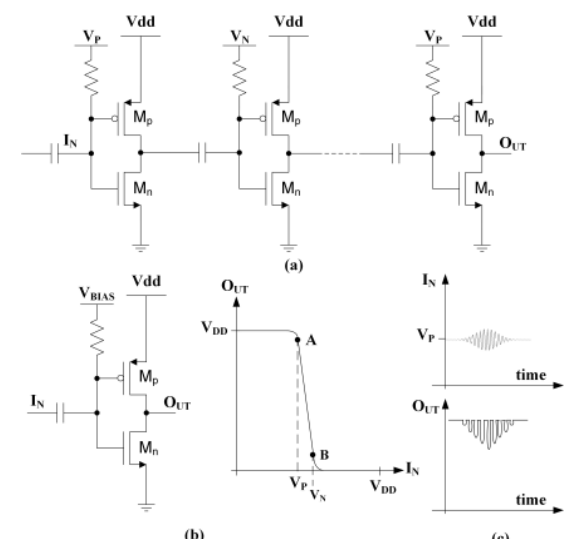

Fig. 10. Rectifier stage: (a) Schematic, (b) CMOS inverter based rectifier cell principle, (c) Simplified waveforms obtained at the rectifier input/output.

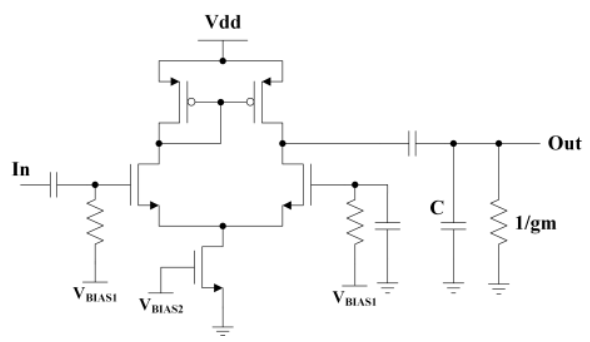

Fig. 11. First order low-pass filter schematic.

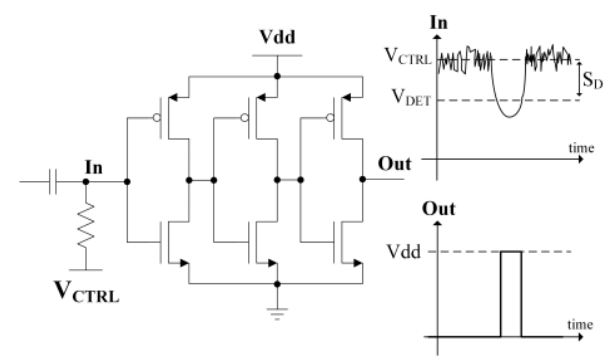

Fig. 12. Decision stage circuit.

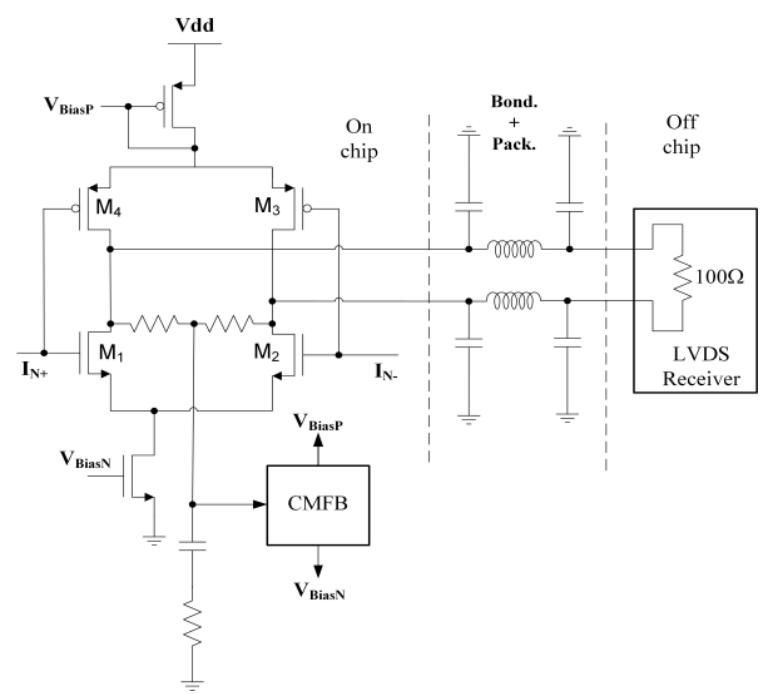

Fig. 13. LVDS transmitter schematic. 


\section{NON-COHERENT RECEIVER DESIGN}

The presented NC IR-UWB receiver shown in Fig. 3 has been designed to be used with $3 \mathrm{GHz}-5 \mathrm{GHz}$ pulses. It consists of a LNA, a voltage peak detector, and a LVDS driver, which are described in this section.

\section{A. LNA}

The LNA architecture is depicted in Fig. 8. The LNA includes three stages: a LC matched input stage and two stages with active load. The LC matched input stage $\left(\mathrm{M}_{1}, \mathrm{M}_{2}\right)$ achieves a bandpass response in the $3-5 \mathrm{GHz}$ bandwidth [26]. In a low-cost perspective, the use of an off-chip filter should be avoided, so the LNA bandpass response reduces both parasitic signals and equivalent noise bandwidth before the pulse detector input. The two active load stages $\left(\mathrm{M}_{3}, \mathrm{M}_{4}\right.$ and $\mathrm{M}_{5}, \mathrm{M}_{6}$ ) provide an additional gain controlled by the voltage $V_{\text {GAIN }}$. The LNA sizing process is done to minimize the noise figure and to maximize the voltage gain as presented in [26]. For such a high gain amplifier at high frequency, signal integrity and stability are important issues. The power supply pads $\left(V_{D D}\right)$ are decoupled by using several MIM and MOS capacitors $\left(C_{D E C}\right.$ in Fig. 8$)$ in order to get a large on chip capacitor value (a few hundred of $\mathrm{pF}$ ) leading to low return path ground impedance at high frequencies.

\section{B. Peak detector}

The proposed peak detector architecture is given in Fig. 9. The incoming UWB pulse is first rectified and a low-pass filter is then used to extract the pulse envelope and to reduce the noise bandwidth. Finally a decision stage based on a comparator followed by a shaping circuit provides a rectangular pulse when pulse envelope crosses the decision voltage $V_{D E T}$.

\section{1) Rectifier stage}

The rectifier is the key element of the detector architecture. A wide bandwidth rectification is achieved by using a biased CMOS inverters chain. Part (b) of Fig. 10 depicts the operation of a biased CMOS inverter. If the biasing voltage $V_{B I A S}$ is set to $V_{P}$, the CMOS inverter operates close to the point A of its characteristic. By using this operating point, the positive part of the input signal is amplified and inverted while the negative part of the input signal is suppressed. Conversely, if $V_{\text {BIAS }}$ is set to $V_{N}$ the point $\mathrm{B}$, the CMOS inverter operates close to the point $B$ of its characteristic. In this case, the negative part of the input signal is amplified and inverted while the positive part of the input signal is suppressed. By cascading CMOS inverters alternatively biased with $V_{P}$ and $V_{N}$ voltages, the input signal is rectified and also amplified. The rectifier stage uses five $\mathrm{AC}$ coupled CMOS inverters alternatively biased with voltage $V_{P}$ and $V_{N}$. Part (c) of Fig. 10 shows the simplified waveforms obtained in the case of an even number of rectifier cells.

\section{2) Low-pass filter}

The low-pass filter consists of three cascaded AC coupled first-order filters with a cutoff frequency of $2 \mathrm{GHz}$. As shown in Fig. 11, the first order low-pass cell is achieved by a singleended transconductance loaded by a capacitor and a resistor. This simple architecture does not allow the filter cutoff frequency to be adjusted, but avoids the implementation of a common mode feedback circuit commonly used in gm-c filters.

\section{3) Decision stage}

As shown in Fig. 12, the decision stage circuit is built from CMOS inverters. The gate of the first inverter is externally biased by a control voltage $V_{C T R L}$ which allows the noise amplitude at its input to be maintained up the switching threshold of the inverter. A set of two other CMOS inverters follows the first one in order to generate a clean logic signal. The amplitude of the UWB pulse envelope is compared to a threshold voltage $V_{D E T}$ at a distance $S_{D}$ of $\mathrm{V}_{C T R L}$. If the pulse envelope crosses the threshold $V_{D E T}$, a pulse is detected and the output signal goes to $V_{D D}$ long enough to enable the postdetection circuits to properly process the signal. The decision threshold value is set to optimize the trade-off between the false alarm rate, due to the influence of noise, and the nondetection rate and so the receiver sensitivity.

\section{LVDS transmitter}

The LVDS interface allows high bit rates (up to $100 \mathrm{Mbs}^{-1}$ ) to be achieved for chip to board interface with a low sensitivity to parasitic capacitors and inductors. The implemented LVDS transmitter circuit uses the typical architecture based on four MOS switches in a bridge configuration as shown in Fig. 13. When the pair (M1, M3) is active and the pair (M2, M4) is off, the polarity of the output current is positive together with the differential output voltage. Conversely, if the pair (M1, M3) is off and the pair (M2, M4) is active, the polarities of the output current and of the differential output voltage are reversed. The required termination resistors are provided by an off-chip standard LVDS receiver chips. With a nominal $100 \Omega$ load on the LVDS receiver side, the differential swing at the output should fall within the LVDS standard specifications $(350 \mathrm{mV})$. Nevertheless, since the supply voltage of the integrated circuit is $1.2 \mathrm{~V}$, the common mode voltage does not match with the LVDS standard specifications $(1.25 \mathrm{~V})$. The common mode voltage is set here at $V_{D D} / 2(0.6 \mathrm{~V})$ by the common mode feedback block and an external chip receiver supporting such a common mode voltage must be used.

\section{Measurement Result}

The emitter and the receiver of the presented transceiver have been realized in two separated chips using the same 130nm CMOS technology from STMicroelectronics and have a supply voltage of $1.2 \mathrm{~V}$. Even if the transmitter could have better performances with a more recent process due to its alldigital architecture, this technological node achieves good performances up to $5 \mathrm{GHz}$ and is a good tradeoff between cost and performances [2]. Emitter and receiver have been integrated on two separated chips, and measured with two different setups for a better test convenience.

\section{A. Transmitter}

As shown on part (a) of Fig. 14, the pulse synthesizer has a 
die area of $1.4 \mathrm{~mm}^{2}$ and a core area of only $0.11 \mathrm{~mm}^{2}$. To measure the pulse synthesizer, it has been packaged into a QFN32 package and three boards (represented in parts (b) of Fig. 17) have been realized: one RF-board to receive the chip, another one to generate the required supply and control voltages and an USB-board to connect with a computer. The test setup principle is given in part (a) of Fig. 17.

To generate a pulse for a given bandwidth with a pulse synthesizer, available values of $A_{n}$ and $\tau_{n}$ have been characterized for the tuning ranges of $V_{a-n}$ and $V_{d-n}$. Next, the analytical pulse model has been used to set all $V_{a-n}$ and $V_{d-n}$ voltages according to the targeted central frequency $f_{M}$ and bandwidth $B W_{-X \mathrm{~dB}}$. Finally, all $V_{a-n}$ are adjusted in order to optimize rejection and also respect FCC requirements. To verify that emitted pulses are well-suited to the targeted bandwidth, or to compensate for spectrum variations generally due to slow phenomenon such as temperature variations, the synthesizer can be controlled by a calibration system as described in [27].

From a Gaussian up-converted pulse with $f_{M}$ and $B W_{-10 d B}$ respectively equal to $4 \mathrm{GHz}$ and $1.8 \mathrm{GHz}$, an UWB pulse has been synthesized by adjusting the control voltages and is shown in part (a) of Fig. 15. Its peak to peak voltage is about $0.9 \mathrm{Vpp}$ and its energy is equal to $0.64 \mathrm{pJ}$ on a $100 \Omega$ load (which is greater than the $0.52 \mathrm{pJ}$ estimated from (8) since some parts of the pulse have a magnitude greater than the model). The delayed pulses needed for RA-OOK signaling are represented in part (b) of Fig. 15.

Due to the gated nature of IR-UWB signals, the mean consumed power can be written as a function of the Pulse Repetition Frequency (PRF) as follows [28] :

$$
P_{C}(P R F)=E_{A C} \cdot P R F+P_{D C}
$$

where $P_{D C}$ is the DC consumed power when no pulse is emitted and $E_{A C}$ is the additional consumed energy when a pulse is emitted. These parameters can be extracted from two powers measured at different PRF which are here for the worst

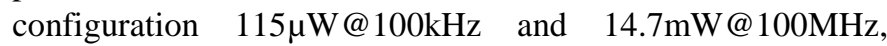
which give a $P_{D C}$ equals to $100 \mu \mathrm{W}$ and an $E_{A C}$ equals to $146 \mathrm{pJ}$ per pulse. At $1 \mathrm{MHz}$, the pulse generator consumes $250 \mu \mathrm{W}$ which gives a $250 \mathrm{pJ}$ energy consumed per pulses.

Finally, the pulse shaping capabilities of the pulse generator are presented in Fig. 16. The targeted $-10 \mathrm{~dB}$ bandwidths, $3.5 \mathrm{GHz}$ (a) and $2.4 \mathrm{GHz}$ (b), are obtained in measurement thanks to the analytical pulse model given in (3). It appears that it is possible to change the central frequency from $3 \mathrm{GHz}$ to $5 \mathrm{GHz}$ by varying the delay $\tau$ in the VCDL. For a pulse centered at $4 \mathrm{GHz}$, the fine tuning of $V_{a-n}$ voltages allows $A_{n}$ to be linearly controlled between $0 \mathrm{~V}$ and $0.45 \mathrm{~V}$ when $V_{a-n}$ is between $0 \mathrm{~V}$ and the supply voltage $1.2 \mathrm{~V}$.

The pulse generator is also able to address several bandwidths and several center frequencies with a high output dynamic as shown in Table I which gives a summary of different reported pulse generators with pulse shaping capabilities. The presented pulse generator achieves a quite

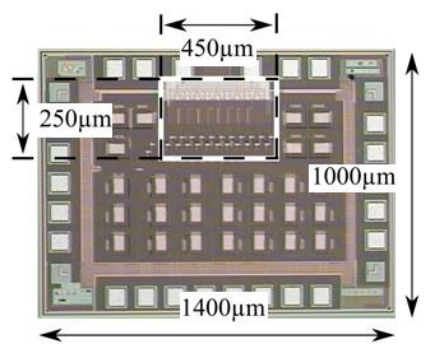

(a)

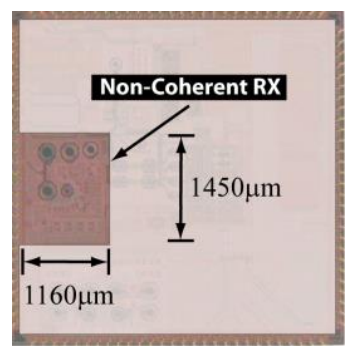

(b)
Fig. 14. Chip micrograph of the proposed pulse generator (a) and of the proposed receiver (b).


(a)

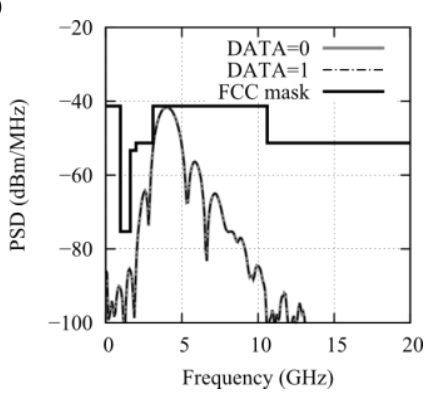

(b)

Fig. 15. Comparison of the ideal Gaussian up-converted pulse with measured transient and frequency responses of a pulse with $A=0.45 \mathrm{~V}, f_{M}=4 \mathrm{GHz}$, and $B W_{-10 \mathrm{~dB}}=1.8 \mathrm{GHz}$ for DATA $=$ ' 0 ' (a). Comparison between pulses required for RA-OOK $($ DATA = ' 1 ' and DATA = '0') $(\mathrm{b})$.
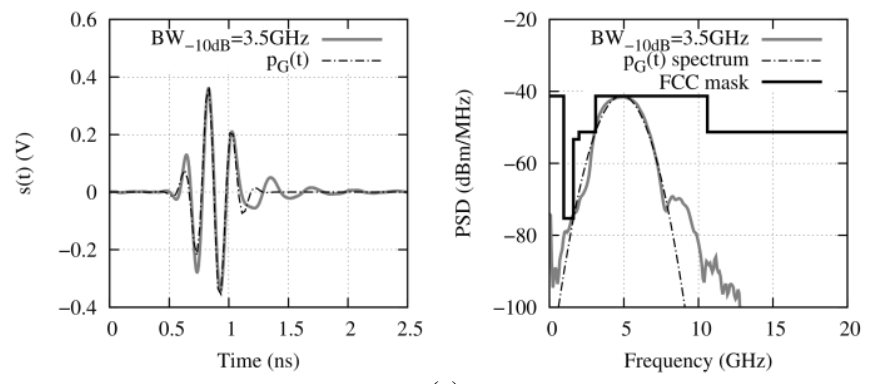

(a)
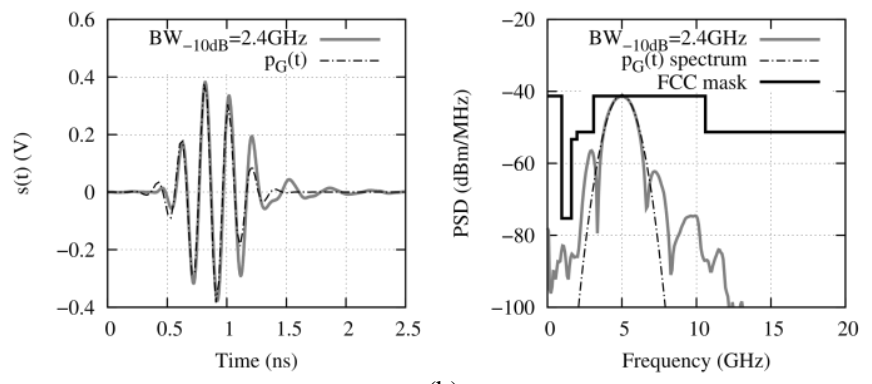

(b)

Fig. 16. Comparison of ideal Gaussian up-converted pulses with measured transient and frequency responses of a $4.85 \mathrm{GHz}$ centered pulse with $\mathrm{BW}_{-10 \mathrm{~dB}}=$ $3.5 \mathrm{GHz}$ (a) and of a $5 \mathrm{GHz}$ centered pulse with $\mathrm{BW}_{-10 \mathrm{dR}}=2.4 \mathrm{GHz}(\mathrm{b})$. 
TABLE I : COMPARISON OF RECENT PULSE GENERATORS WITH PULSE SHAPING CAPABILITIES

\begin{tabular}{|c|c|c|c|c|c|c|c|}
\hline$[\mathrm{REF}]$ & [9] & {$[11]$} & {$[16]$} & [17] & [32] & [33] & This Work \\
\hline $\mathrm{V}_{\mathrm{DD}}(\mathrm{V})$ & 0.9 & 1 & 1 & 0.9 & 1.2 & 1.2 & 1.2 \\
\hline $\mathrm{f}_{\mathrm{M}}(\mathrm{GHz})$ & $3.5-4.5$ & $3-5$ & 4 & $3.1-5$ & $3.1-6$ & 4.9 & $3-5$ \\
\hline $\mathrm{BW}_{-10 \mathrm{~dB}}(\mathrm{GHz})$ & 0.5 & 0.5 & 2 & $0.5-1.4$ & $1-1.86$ & 3.5 & $1.8-3.5$ \\
\hline$A(V)$ & 0.25 & 0.305 & 0.25 & $0.048-0.063$ & 0.2 & 0.11 & 0.45 \\
\hline $\mathrm{E}_{\mathrm{AC}}(\mathrm{pJ} / \mathrm{pulse})$ & N/A & 2 & N/A & $8-12$ & N/A & N/A & 146 \\
\hline$P_{D C}(\mu W)$ & 2.8 & 105 & 28 & 170 & N/A & N/A & 100 \\
\hline $\mathrm{P}_{\mathrm{C}} @ \mathrm{PRF}(\mathrm{mW})$ & 5.7 & 4.36 & N/A & 0.57 & N/A & 87.6 & 14.7 \\
\hline PRF (MHz) & 0.1 & 15.6 & 32 & 50 & $<500$ & 800 & 100 \\
\hline $\mathrm{E}_{\mathrm{C}}(\mathrm{nJ} / \mathrm{pulses})$ & 0.057 & 0.28 & 0.008 & 0.012 & $0.0036-0.02$ & 0.1 & 0.147 \\
\hline Tech. (CMOS) & $90 \mathrm{~nm}$ & $90 \mathrm{~nm}$ & $65 \mathrm{~nm}$ & $90 \mathrm{~nm}$ & $90 \mathrm{~nm}$ & $130 \mathrm{~nm}$ & $130 \mathrm{~nm}$ \\
\hline
\end{tabular}

good output dynamic $(A=0.45 \mathrm{~V})$ compared to other reported works. The tradeoff between output dynamic and power consumption is comparable to other all-digital synthesizers, which generally consume more power than analog pulse generators as it can be observed in Table I.

\section{B. Receiver}

The non-coherent receiver core area is $1.7 \mathrm{~mm}^{2}$ as indicated in part (b) of Fig. 14. The chip where the receiver has been integrated includes other circuits which are out of the scope of this paper. It has been packaged in a QFN88 package. The DC power consumption of the non-coherent receiver is $30.5 \mathrm{~mW}$. From post-layout simulations, the LNA achieves for the $3.1 \mathrm{GHz}-4.9 \mathrm{GHz}$ band, a minimum voltage gain of $28 \mathrm{~dB}$, a maximum noise figure of $3 \mathrm{~dB}$, a maximum $\mathrm{S} 11$ of $-11.5 \mathrm{~dB}$, and a current consumption of $17 \mathrm{~mA}$.

The performance of the non-coherent receiver has been measured through the BER characteristic. As it can be seen in part (c) of Fig. 17, three boards have been realized, one RFboard to receive the chip, another one to generate all the required supply voltages and control signals and a PXI-board for connection with a PC. A $2 \mathrm{GHz}$ bandwidth pulse centered at $4 \mathrm{GHz}$ is used for the measurement setup. The measurement has been done using the same considerations than in the theoretical BER calculus of the section II.D: a sequence of successive ' 1 ' is sent to measure the probability of a nondetection and a sequence of successive ' 0 ' is sent to measure the probability of a false-alarm. The detection threshold is set to obtain the same number of false-alarms and non-detections. The number of successive ' 1 ' and successive ' 0 ' used for this measurement is $10^{6}$.The measurement has been done for a rate of $15.6 \mathrm{Mbps}$ and the receiver exhibits a $10^{-3} \mathrm{BER}$ for a pulse energy of 5.35 aJ. This corresponds to a $-85.8 \mathrm{dBm}$ sensitivity at a $1 \mathrm{Mbps}$ rate for $\mathrm{OOK}$ modulations. This measured sensitivity value is much lower than the theoretical one presented in Fig. 4 (-98 dBm if downscaled to $1 \mathrm{Mbps})$ since Fig. 4 gives the upper performance limit for a receiver using ideal blocks with no noise, no loss, and no bandwidth limitation. However, this sensitivity is comparable to the performances of other receivers based on energy detection.

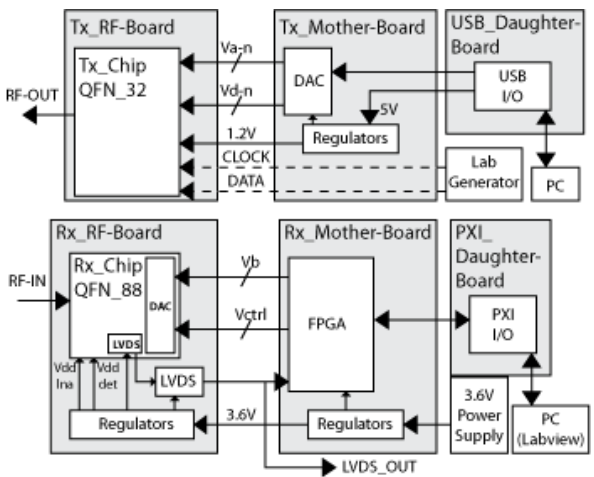

(a)

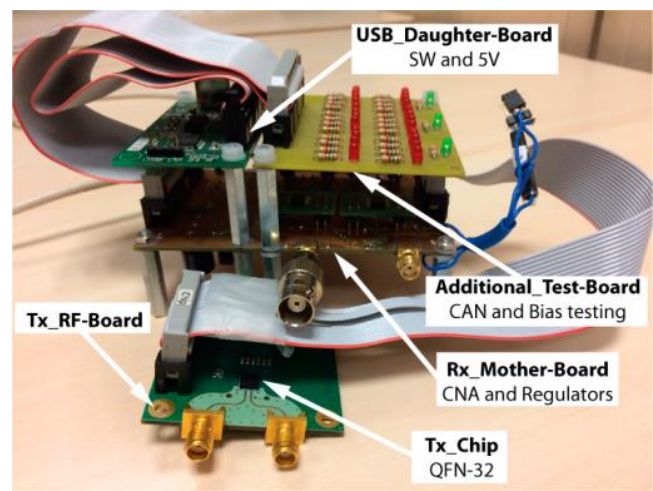

(b)

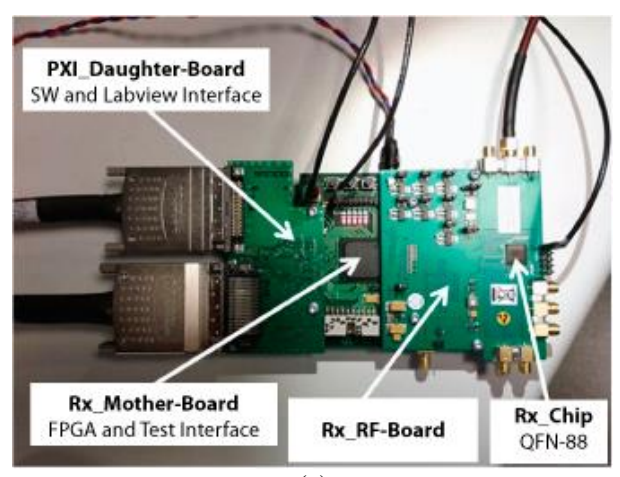

(c)

Fig. 17. Measurement setup principle (a), emitter measurement setup (b) receiver measurement setup (c). 


\begin{tabular}{cc}
\hline \multicolumn{2}{c}{ TABLE II : FINAL LINK BUDGET } \\
\hline $\mathrm{PRF}_{\mathrm{MEAN}}(\mathrm{MHz})$ & 0.5 \\
$\mathrm{D}_{\mathrm{b}}(\mathrm{Mbps})$ & 1 \\
$\mathrm{BW}_{-10 \mathrm{~dB}}(\mathrm{GHz})$ & 1.8 \\
$\mathrm{E}_{\mathrm{P}}(\mathrm{pJ})$ & $0.52^{* * *}$ \\
$\mathrm{~A}(\mathrm{~V}) * *$ & 0.45 \\
$\mathrm{~A}_{\mathrm{RX}}(\mathrm{mV})^{*}$ & 1.1 \\
$\mathrm{E}_{\mathrm{RX}}(\mathrm{pJ})$ & $5.35 \cdot 10^{-6}$ \\
$\mathrm{~S}_{\mathrm{RX}} @ 1 \mathrm{Mbps}(\mathrm{dBm})$ & -85.8 \\
$\mathrm{~d}(\mathrm{~m})$ & 1.9 \\
*on a $\overline{\text { 50 } \Omega \text { impedance }}$ \\
**on a $100 \Omega$ impedance \\
*** estimated from $(8)$ \\
C. Overview
\end{tabular}

The final budget link based on measurement results is presented in Table II and introduces $A_{R X}$ (resp. $E_{R X}$ ) which is the peak magnitude (resp. energy) of the received pulse, and $S_{R X}$ which is the receiver sensitivity for a given bit rate. By using the emitted pulse indicated in Fig. 15 and the measured receiver sensitivity, the range $d$ is computed from Friis formula. Thus, the maximum communication range is estimated to $1.9 \mathrm{~m}$ with the radiated pulse energy on a $100 \Omega$ differential load and the receiver sensitivity on a $50 \Omega$ load. In this budget link, no antenna gain and no fade margin are taken into account. This budget link shows that peak voltage detection is suitable for short range communications. The transceiver performances are compared with previous works in table III. This shows that peak voltage detection exhibits performances comparable to other type of detectors. The high output voltage of the transmitter combined with the receiver sensitivity finally gives a $1.9 \mathrm{~m}$ communication range which is at the state of the art.

\section{CONCLUSION}

A 3-5 GHz peak voltage detector based IR-UWB CMOS transceiver with pulse shaping capabilities has been presented in a 130nm CMOS technology. A non-coherent receiver based on a peak power detector has been presented. The detection is fully asynchronous. The receiver has a sensitivity of $-85.8 \mathrm{dBm}$ for a rate of $1 \mathrm{Mbps}$. This sensitivity allows detecting an input signal with a magnitude of $1.1 \mathrm{mV}$ which leads to a communication range of $1.9 \mathrm{~m}$. The receiver power consumption is about $30.5 \mathrm{~mW} @ 100 \mathrm{Mbps}$. The transmitter emits pulses with a high pulse shaping capabilities for a power consumption of 14.7mW@100Mbps.

\section{APPENDIX}

In this appendix, an estimation of the BER which can be achieved with an UWB energy detector is presented by considering an OOK coding, an AWGN channel, and no multipath propagation. The considered receiver architecture is similar to the one depicted in Fig. 3 but the peak voltage detector has been replaced by a perfect energy detector. This energy detector collects without any loss the signal energy available at the LNA output during the integration window. A perfect synchronization is assumed for the integration window.

\section{A. Probability of error}

During the integration window $T_{S}$, the continuous time domain signal at the receiver input is replaced by $P$ discrete samples of the received signal obtained at the Nyquist rate:

$$
P=2 \cdot f_{\text {MAX }} \cdot T_{S}+1 \text {. }
$$

Given that, for the $3.1 \mathrm{GHz}-4.9 \mathrm{GHz}$ signal bandwidth considered here, the noise bandwidth $(1.8 \mathrm{GHz})$ is close to the maximum spectrum frequency $f_{M A X}(4.9 \mathrm{GHz})$, it is assumed that the noise samples are independent. Consequently, the

TABLE III PERFORMANCE COMPARISON WITH PREVIOUS WORKS

\begin{tabular}{|c|c|c|c|c|c|c|c|c|c|c|c|c|c|}
\hline Ref & Year & $\begin{array}{c}\text { Tech. } \\
\text { (CMOS) }\end{array}$ & $\begin{array}{c}\text { size } \\
\left(\mathbf{m m}^{2}\right)\end{array}$ & $\begin{array}{c}\text { Pulse } \\
\text { BW } \\
(\mathbf{G H z})\end{array}$ & $\begin{array}{c}\mathbf{f}_{\mathbf{M}} \\
(\mathbf{G H z})\end{array}$ & $\begin{array}{c}\text { d } \\
(\mathbf{m})\end{array}$ & $\begin{array}{c}\text { Data Rate } \\
\text { (bps) }\end{array}$ & $\begin{array}{c}\text { Tx Power } \\
\text { Cons. at } \\
\text { Data Rate } \\
(\mathbf{m W})\end{array}$ & $\begin{array}{c}\text { Tx cons. } \\
\text { nrj/b } \\
(\mathbf{p J} / \mathbf{b})\end{array}$ & $\begin{array}{c}\text { Rx Sensi. } \\
\text { at Data } \\
\text { Rate } \\
(\text { dBm })\end{array}$ & $\begin{array}{c}\text { Rx Sensi. } \\
\text { Scaled } \\
\text { @ 1Mbps } \\
\text { (dBm) }\end{array}$ & $\begin{array}{c}\text { Rx Power } \\
\text { Cons. at } \\
\text { Data Rate } \\
(\mathbf{m W})\end{array}$ & $\begin{array}{c}\text { Rx cons. } \\
\text { nrj/b } \\
(\mathbf{n J} / \mathbf{b})\end{array}$ \\
\hline This Work & & $130 \mathrm{~nm}$ & 1.31 & $0.5-2$ & $3.5-5$ & 1.9 & $100 \mathrm{M}$ & 14.7 & 147 & -65.8 & -85.8 & 30.5 & 0.305 \\
\hline [3] & $\underline{2013}$ & $90 \mathrm{~nm}$ & 1.7 & 0.5 & $3.5-4.5$ & 0.5 & $100 \mathrm{k}$ & 0.0085 & 60.7 & -85 & -75 & 0.11 & 0.8 \\
\hline [16] & $\underline{2015}$ & $65 \mathrm{~nm}$ & 2.25 & 0.5 & $7.5-9.5$ & 1.2 & $500 \mathrm{M}$ & 6.9 & 13.8 & -60 & -87 & 5.9 & 0.0118 \\
\hline [29] & $\underline{2011}$ & $90 \mathrm{~nm}$ & 0.6 & $3.6-4.3$ & $2.9-3.8$ & 1.5 & $1 \mathrm{M}$ & 0.258 & 258 & $-60 /-66$ & $-60 /-66$ & $1.64-2.18$ & 1.64 \\
\hline [30] & $\underline{2014}$ & $180 \mathrm{~nm}$ & 4.4 & 1.36 & $3-5$ & N/A & $20 \mathrm{M}$ & 13.4 & 670 & -81 & -94 & $3.54 *$ & 3.54 \\
\hline [31] & $\underline{2016}$ & $65 \mathrm{~nm}$ & $4.6^{* *}$ & 0.5 & $3.1-10.6$ & 1 & $1 \mathrm{G}$ & $31.9^{* * *}$ & 31.9 & -74 & -104 & $27.8^{* * *}$ & 0.0278 \\
\hline [34] & $\underline{2008}$ & $180 \mathrm{~nm}$ & 0.36 & 1 & 3 & 0.04 & $500 \mathrm{M}$ & 0.28 & 0.56 & N/A & N/A & 11 & 0.022 \\
\hline [35] & $\underline{2013}$ & $180 \mathrm{~nm}$ & 2.73 & N/A & $9-12$ & N/A & $30 \mathrm{k}$ & 0.022 & 747 & -77 & -61.7 & 0.037 & 1.2 \\
\hline
\end{tabular}

*at $1 \mathrm{Mbps}$, **with pads, ***with PLL 
discrete samples of the received signal are the sum of samples of the unnoisy signal and samples of the noise.

Assuming an AWGN channel and the independence of the noise samples, the probability density function of the noise voltage for each sample is given by:

$$
p_{N}(v)=\frac{1}{\sqrt{2 \pi \sigma_{N}^{2}}} \exp \left(\frac{-v^{2}}{2 \sigma_{N}^{2}}\right)
$$

Assuming that the noise bandwidth is the same that the bandwidth $(B W)$ of the signal, the noise variance $\sigma_{N}$ at the LNA output is given by:

$$
10 \log \left(\frac{\sigma_{N}^{2}}{R_{L N A}}\right)=10 \log (K \cdot T \cdot B W)+G_{L N A}+N F_{L N A}
$$

where $K$ is the Boltzmann constant, $T$ the absolute temperature, $R_{L N A}, G_{L N A}$, and $N F_{L N A}$ the input resistor, the gain and the noise figure of the LNA respectively.

For an OOK coding, the probability of error is given by:

$$
\operatorname{Pr}_{E}=0.5 \times \operatorname{Pr}_{F A}+0.5 \times \operatorname{Pr}_{N D}
$$

where $\operatorname{Pr}_{\mathrm{FA}}$ is the probability of a false alarm and $\operatorname{Pr}_{\mathrm{ND}}$ is the probability of a non-detection.

\section{B. Probability of non-detection}

A non-detection occurs when the energy of the noisy signal integrated during the integration window $T_{S}$ remains lower than the decision threshold. The energy collected by the energy detector is given by:

$$
W=\frac{T_{S}}{R \cdot P} \sum_{P}\left(S_{i}+N_{i}\right)^{2}
$$

where $S_{i}$ are the discrete samples of the unnoisy signal, $N_{i}$ the discrete samples of the noise, and $R$ is the input impedance of the energy detector which is assumed to be real.

The sum of the discrete sample of the signal $S_{i}$ and of the noise $N_{i}$ is a random variable $X_{i}$ :

$$
X_{i}=S_{i}+N_{i}
$$

The $P$ values of $X_{i}$ are $P$ (independent) normally distributed random variables with a mean equal to the corresponding unnoisy signal sample and a variance equal to the noise variance $\sigma_{N}$. Consequently the random variable $Y_{i}$ defined as:

$$
Y_{i}=\frac{X_{i}}{\sigma_{N}}
$$

has a unit variance and:

$$
Y=\sum_{i=1}^{P} Y_{i}^{2}=\sum_{i=1}^{P} \frac{X_{i}^{2}}{\sigma_{N}^{2}}
$$

is a random variable which is distributed according to the noncentral chi-squared distribution. The relation between $Y$ and the energy $(W)$ collected by the energy detector during $T_{S}$ can be written as follows:

$$
W=\frac{T_{S} \cdot \sigma_{N}^{2}}{R \cdot P} Y
$$

$Y$ has two parameters: $P$ the number of samples which specifies the number of degrees of freedom, and $\lambda$ which is related to the mean of the random variables by:

$$
\lambda=\sum_{P}\left(\frac{S_{i}}{\sigma_{N}}\right)^{2}=\frac{R \cdot P}{T_{S} \cdot \sigma_{N}^{2}} W_{S}
$$

where $W_{S}$ is the energy of the unnoisy signal. (21) leads to:

$$
W_{S}=\frac{T_{S}}{R \cdot P} \sum_{P} S_{i}^{2}
$$

The probability density function of $Y$ is given by:

$$
f_{Y}(x, P, \lambda)=\frac{1}{2} e^{-(x+\lambda) / 2}\left(\frac{x}{\lambda}\right)^{P / 4-1 / 2} I_{P / 2-1}(\sqrt{\lambda x})
$$

where $I_{\alpha}(y)$ is $\alpha$ order modified first kind Bessel function which is given by:

$$
I_{\alpha}(y)=\sum_{j=0}^{\infty} \frac{(y / 2)^{2 j+\alpha}}{j ! \Gamma(\alpha+j+1)}
$$

with the Gamma function $\Gamma(\mathrm{z})$ defined as follows:

$$
\Gamma(z)=\int_{0}^{\infty} x^{z-1} e^{-x} d x
$$

Finally the cumulative distribution function of $Y$ is given by:

$$
F_{Y}(x, P, \lambda)=\sum_{j=0}^{\infty} e^{-\lambda / 2} \frac{(\lambda / 2)^{j}}{j !} Q(x, P+2 j)
$$

where $Q(x, k)$ is the cumulative distribution function of the central chi-squared distribution with $k$ degrees of freedom, which is given by:

$$
Q(x, k)=\frac{\gamma(k / 2, x / 2)}{\Gamma(k / 2)}
$$

where $\gamma(z, a)$ is the lower incomplete Gamma function defined as follows:

$$
\gamma(z, a)=\int_{0}^{a} x^{z-1} e^{-x} d x .
$$

A non-detection occurs when the energy collected by the energy detector is lower than the decision threshold $W_{D}$. It occurs when:

$$
\frac{T_{S} \cdot \sigma_{N}^{2} \cdot Y}{R \cdot P}<W_{D}
$$

which leads to:

$$
Y<\frac{R \cdot P \cdot W_{D}}{T_{S} \cdot \sigma_{N}^{2}}
$$

So the probability of a non-detection can be obtained by using the cumulative distribution of $Y$ : 


$$
\operatorname{Pr}_{N D}=F_{Y}\left(\frac{R \cdot P \cdot W_{D}}{T_{S} \cdot \sigma_{N}^{2}}, P, \frac{R \cdot P \cdot W_{S}}{T_{S} \cdot \sigma_{N}^{2}}\right)
$$

\section{Probability of false alarm}

A false alarm occurs when the energy collected is greater than the decision threshold $W_{D}$ while no pulse has been sent during the integration window. The mathematical expressions given in the previous part are not valid in this case because when no pulse has been sent, the parameter $\lambda$ defined in (21) is equal to zero. In the case where $\lambda$ is equal to zero, the random variable $Y$ defined in (18) becomes a normal law which is called $Y^{\prime}$ in the following. Thus, $Y^{\prime}$ is a random variable which is distributed according to the central chisquared distribution with $P$ degrees of freedom and with a probability density function given by:

$$
f_{Y^{\prime}}(x, P)=\frac{x^{P / 2-1} e^{-x / 2}}{2^{P / 2} \Gamma(P / 2)} .
$$

The cumulative distribution function of $Y^{\prime}$ is also given by:

$$
F_{Y^{\prime}}(x, P)=\frac{\gamma(P / 2, x / 2)}{\Gamma(P / 2)} .
$$

The energy collected $W$ by the energy detector during the integration window can be computed and is given by:

$$
W=\frac{T_{S} \cdot \sigma_{N}^{2}}{R \cdot P} Y^{\prime}
$$

A false-alarm occurs when the energy collected by the energy detector given by $W$ is greater than the decision threshold $W_{D}$. It occurs when:

$$
\frac{T_{S} \cdot \sigma_{N}^{2}}{R \cdot P} Y^{\prime} \geq W_{D}
$$

which leads to:

$$
Y^{\prime} \geq \frac{R \cdot P \cdot W_{D}}{T_{S} \cdot \sigma_{N}^{2}}
$$

So the probability of a false alarm can be obtained by using the cumulative distribution of $Y^{\prime}$ :

$$
\operatorname{Pr}_{F A}=1-F_{Y^{\prime}}\left(\frac{R \cdot P \cdot W_{D}}{T_{S} \cdot \sigma_{N}^{2}}, P\right) .
$$

\section{BER Calculation}

Knowing the probability of a non-detection given by (31) and the probability of a false-alarm given by (37), (12) gives the probability of error for a given received power, a given integration window and a given decision threshold. Similarly as in the case of a peak voltage detection, there is an optimal decision threshold $W_{D}$ that minimizes the received power and balances $\operatorname{Pr}_{F A}$ and $\operatorname{Pr}_{N D}$. So, by using relations (12), (31), (37) and an iterative algorithm, the receiver sensitivity can be obtained for a given BER. Finally, the use of Friis formula with this minimum received input power gives the achievable range for a given emitted power. By using the UWB pulse defined by (3) with a $-10 \mathrm{~dB}$ bandwidth of $1.8 \mathrm{GHz}$ centered at $4 \mathrm{GHz}$, the theoretical BER obtained with an energy detector is given in Fig. 4 for a date rate of 100Mbps with OOK encoding, an integration window of $2 \mathrm{~ns}$, and a LNA noise figure of $3 \mathrm{~dB}$.

\section{REFERENCES}

[1] R. K. Dokania, X. Y. Wang, S. G. Tallur, and A. B. Apsel, "A Low Power Impulse Radio Design for Body-Area-Networks," IEEE Trans. Circuits and Systems I, vol. 58, no. 7, pp. 1458-1469, 2011.

[2] D. Morche, G. Masson, S. De Rivaz, F. Dehmas, S. Paquelet, A. Bisaux, O. Fourquin, J. Gaubert, S. Bourdel, "A Double Quadrature UWB Receiver for wide Range Localization Applications with sub-cm Precision," IEEE Journal of Solid State Circuit, Volume 48, Issue 10, pp. 2351-2362, 2014.

[3] X. Y. Wang, R. K. Dokania, A. B. Apsel, "A Crystal-Less SelfSynchronized Bit-Level Duty-Cycled IR-UWB Transceiver System," IEEE Transaction on Circuits and Systems I: Regular Papers, vol. 66, no. 9, pp. 2488-2501, 2013.

[4] B. Vigraham, P. R. Kinget, "A Self-Duty-Cycled and Synchronized UWB Pulse-Radio Receiver SoC With Automatic Threshold-Recovery Based Demodulation," IEEE Journal of Solid-State Circuits, vol. 49, no. 3, pp. 581-594, 2014.

[5] M. Crepaldi, S. Macis, P. M. Ros, D. Demarchi, "A 0.07 mm Asynchronous Logic CMOS Pulsed Receiver Based on Radio Events Self-Synchronization," IEEE Transactions on Circuits and Systems I: Regular Papers, vol. 61, no. 3, pp.750-763, 2014.

[6] O. Ramos Sparrow, R. Vauche, N. Dehaese, S. Bourdel, J. Gaubert, I. Ben Amor, E. Muhr, P. Losco and O. Fourquin, "High rate UWB CMOS transceiver chipset for WBAN and biomedical applications," Analog Integrated Circuits and Signal Processing, vol. 81, no. 1, pp. 215-227, 2014.

[7] S. Bourdel, Y. Bachelet, J. Gaubert, R. Vauche, O. Fourquin, N. Dehease, N. \& H. Barthelemy, "A 9pJ/Pulse 1.42Vpp OOK CMOS UWB Pulse Generator for the 3.1-10.6 GHz FCC Band," IEEE Transaction on Microwave Theory and Techniques, vol. 58, no. 1, pp. 65-73, 2011.

[8] H. Kim and Y. Joo, "Fifth-Derivative Gaussian Pulse Generator for UWB System," in Proc. Digest of Papers IEEE Radio Frequency integrated Circuits Symposium (RFIC), pp. 671-674, 2005.

[9] R. Dokania, X. Wang, C. Dorta-Quinones, W. Godycki, S. Tallur, A. Apsel, "A $6 \mu \mathrm{W}, 100 \mathrm{Kbps}, 3-5 \mathrm{GHz}$, UWB impulse radio transmitter," in Proc. ACM/IEEE International Symposium on Low-Power Electronics and Design (ISLPED), pp. 91-94, 2010.

[10] T. Norimatsu, R. Fujiwara, M. Kokubo, M. Miyazaki, A. Maeki, Y. Ogata, S. Kobayashi, N. Koshizuka, K. Sakamura, "A UWB-IR Transmitter With Digitally Controlled Pulse Generator," IEEE Journal of Solid-State Circuits, vol. 42, no. 6, June 2007, pp. 1300-1309, 2007.

[11] P. Mercier, D. C. Daly and A. P. Chandrakasan, "An Energy-Efficient All-Digital UWB Transmitter Employing Dual Capacitively-Coupled Pulse-Shaping Drivers," IEEE Journal of Solid-State Circuits, vol. 44, no. 6, pp. 1679-1688, 2009.

[12] S. Bourdel Y. Bachelet, J. Gaubert, M. Battista, M. Egels, N. Dehaese; "Low-cost CMOS pulse generator for UWB systems," Electronics Letters, vol. 43, no. 25, pp. 1425-1427, 2007.

[13] M. Pelissier, D. Morche, and P. Vincent, "Super-Regenerative Architecture for UWB PulseDetection: From Theory to RF Front-End esign," IEEE Transaction on Circuits and Systems I: Regular Papers, vol. 56, no. 7, pp. 1500-1512, 2009.

[14] F. S. Lee and A. P. Chandrakasan, "A 2.5 nJ/bit 0.65 V Pulsed UWB Receiver in $90 \mathrm{~nm}$ CMOS," IEEE Journal of Solid State Circuit, vol. 42, no. 12, pp. 2851-2859, 2007.

[15] R. Dokania, X. Wang, S. Tallur, C. Dorta-Quinones, A. Apsel, "An Ultralow-Power Dual-Band UWB Impulse Radio," IEEE Transactions on Circuits and Systems II: Express Briefs, vol. 57, no. 7, pp. 541-545, 2010.

[16] S. Geng, D. Liu, Y. Li, H. Zhuo, W. Rhee, Z. Wang, "A 13.3 mW 500 $\mathrm{Mb} / \mathrm{s}$ IR-UWB Transceiver With Link Margin Enhancement Technique for Meter-Range Communications," IEEE Journal of Solid-State Circuits, vol. 50, no. 3, pp. 669-678, 2015. 
[17] Y. Park, D. D. Wentzloff, “An All-Digital 12pJ/Pulse IR-UWB Transmitter Synthesized from a Standard Cell Library," IEEE Journal of Solid-State Circuits, vol. 46, no. 5, pp. 1147-1157, 2011.

[18] D. D. Wentzloff and A. P. Chandrakasan, "Delay-based BPSK for pulsed-UWB communication," in Proc. IEEE Int. Conf. Acoustics, Speech and Signal Processing (ICASSP), vol. 3, pp. 561-564, 2007.

[19] H. Kim and Y. Joo, "Fifth-Derivative Gaussian Pulse Generator for UWB System," in Proc. Digest of Papers IEEE Radio Frequency Integrated Circuits Symposium (RFIC), pp. 671-674, 2005.

[20] L. C. Moreira, D. M. Silveira, W. A. M. van Noije, and S. T. Kofuji, “A 5th Derivative Gaussian Pulse CMOS IR-UWB Generator using a Phase Detector," in Proc. German Microwave Conf., pp. 154-157, 2010.

[21] Z. N. Low, J. H. Cheong, and C. L. Law, "Novel Low Cost Higher Order Derivitive Gaussian Pulse Generator Circuit," in Proc. Ninth Int. Conf. Communications Systems (ICCS), pp. 30-34, 2004.

[22] T.-A. Phan, V. Krizhanovskii, S.-K. Han, S.-G. Lee, H. seo Oh, and N.S. Kim, "4.7pj/pulse 7th Derivative Gaussian Pulse Generator for Impulse Radio UWB," IEEE International Symposium on Circuits and Systems (ISCAS), pp. 3043-3046, 2007.

[23] H. L. Van Trees, Detection, Estimation, and Modulation Theory, part $I$. New York: Wiley, 1968.

[24] P. A. Humblet, M. Azizoglu, "On the bit error rate of lightwave systems with optical amplifiers," Journal of Lightwave Technology, vol. 9, no. 11, pp. 1576-1582, 1991.

[25] R. Vauche, S. Bourdel, N. Dehaese, O. Fourquin, and J. Gaubert, "Fully tunable UWB pulse generator with zero DC power consumption," International Conference on Ultra-Wideband (ICUWB), pp. 418-422, 2009.

[26] M. Battista, J. Gaubert, M. Egels, S. Bourdel, H. Barthelemy, "6-10 GHz Ultra Wide-Band CMOS LNA," Electronics Letters, vol. 44, no. 5, pp. 343-344, 2008.

[27] A. Goavec, M. Zarudniev, R. Vauché, F. Hameau, J. Gaubert, E. Mercier, "An Efficient Method of Power Spectral Density Estimation for On-Chip IR-UWB Transmitter Self-Calibration," IEEE Transaction on Circuits and Systems I: Regular Papers, no. 99, pp. 1-10, 2016.

[28] R. Vauche, E. Bergeret, J. Gaubert, S. Bourdel, O. Fourquin, N. Dehaese, "A remotely UHF powered UWB transmitter for high precision localization of RFID tag," IEEE International Conference on Ultra-WideBand (ICUWB), pp. 494-498, 2011.

[29] M. Crepaldi, C. Li, J. R. Fernandes, P. R. Kinget, "An Ultra-Wideband Impulse-Radio Transceiver Chipset Using Synchronized-OOK Modulation," IEEE Journal of Solid-State Circuits, vol. 46 , no. 10, pp. 2284-2299, 2011.

[30] Y. Zheng, Y. Zhu, C-W. Ang, Y. Gao,C-H. Heng, "A 3.54 nJ/bit-RX, $0.671 \mathrm{~nJ} / \mathrm{bit}-\mathrm{TX}$ Burst Mode Super-Regenerative UWB Transceiver in 0.18- CMOS," IEEE Transaction on Circuits and Systems I: Regular Papers, vol. 61, no. 8, pp. 2473-2481, 2014.

[31] N-S. Kim, J. M. Rabaey, "A High Data-Rate Energy-Efficient TripleChannel UWB-Based Cognitive Radio," IEEE Journal of Solid-State Circuits, vol. 51, no. 43, pp.809-820, 2016.

[32] A. Ebrazcl and P. Mohseni, "An all-digital IR-UWB transmitter with a waveform-synthesis pulse generator in 90nm CMOS for high-density brain monitoring," in Proc. Radio Frequency Integrated Circuits Symposium (RFIC), 2013 IEEE, pp. 13-16, June 2013.

[33] S. Bourdel, R. Vauche, O. Ramos, E. Muhr, J. Gaubert, N. Dehaese, and H. Barthelemy, "An inductorless CMOS UWB pulse generator with active pulse shaping circuit," IEEE International Conference on UltraWideband, ICUWB 2013, pp. 175-179, 2013.

[34] M. Sasaki, "A 12-mW 500-Mb/s 1.8- $\mu \mathrm{m}$ CMOS pulsed UWB transceiver suitable for sub-meter short-range wireless communication," in Proc. Radio Frequency Integrated Circuits Symposium, pp. 593-596, 2008.

[35] K. K. Huang, J. K. Brown, E. Ansari, R. R. Rogel, Y. Lee, H. Kim, D. D. Wentzloff, "An Ultra-Low-Power $9.8 \mathrm{GHz}$ Crystal-Less UWB Transceiver With Digital Baseband Integrated in $0.18 \mu \mathrm{m}$ BiCMOS," IEEE Journal of Solid-State Circuits, vol. 48, no. 12, pp. 3178-3189, 2013.

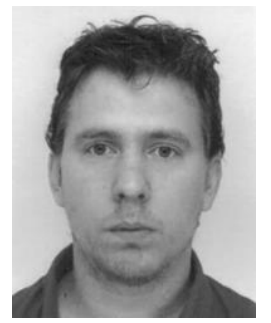

Remy Vauche received his M.Eng. degree in Microelectronics \& Telecommunication from Polytech' Marseille, and the M.S. degree in Microelectronics and Nanoelectronics from Aix-Marseille University, Marseille France, in 2008. He received the Ph.D. degree in Microelectronics from the University of Provence, Aix-Marseille I, France, in 2011. From 2011 to 2014, he was a lecturer and a researcher in the ISEN French Engineering School, Toulon, France. Since 2014, he is now an Associate Professor of the Aix-Marseille University, Marseille, France, and a member of the Integrated Circuits Design Team from the Provence Nanosciences Microelectronics and Materials Laboratory (IM2NP), Marseille, France. His current field of research is in the design of IR-UWB ICs for low-power and low-cost applications.

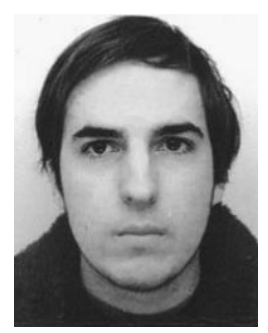

Eloi Muhr was born in Reims, France, in 1989. He received the M.S. degree in microelectronics and nanoelectronics from Provence University, Marseille France, in 2012. He received the Ph.D degree in microelectronics from University of Aix Marseille, France in 2016. Since 2016, he is a member of RFID Team from the Provence Nanoscience Microelectronics and Materials Laboratory (IM2NP), Marseille, France. His current field of research is in the design of analog/RF CMOS integrated circuit for low-cost applications.

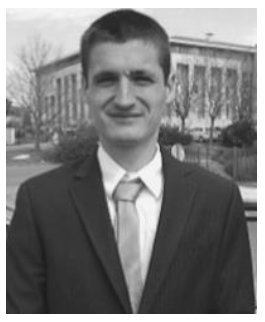

Olivier Fourquin Olivier Fourquin was born in Marseille in 1985. He received a M. Eng degree in Microelectronics and Telecommunications from Polytech' Marseille and a M.S. in Microelectronics and Nanoelectronics from Aix-Marseille University, Marseilles France, in 2008. He received a Ph.D. degree in Microelectronics from Aix-Marseille University in 2011. He currently works at INVIA, Meyreuil, France.

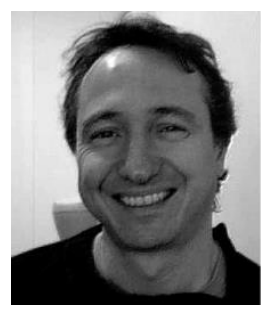

Sylvain Bourdel received the $\mathrm{Ph} . \mathrm{D}$ in microelectronics from the National Institute of Applied Science (INSA) of Toulouse in 2000. He was with the LAAS laboratory of Toulouse where he was involved on radiofrequency systems modelling and he was particularly focused on spread spectrum techniques applied to $2.45 \mathrm{GHz}$ transceivers. In 2002 he joined the IM2NP in Marseille where he headed with $\operatorname{Pr} \mathrm{H}$. Barthélemy the Integrated Circuit Design Team of the IM2NP. He joined in 2013 the Grenoble-INP as a full Professor where he works at the IMEP-LAHC laboratory. He works on RF and MMW IC design and integration. He particularly focuses on low cost and low power applications. His area of interest also includes system level specifications, UWB and RFID. He is the author and co-author of more than 70 referenced IEEE publications. 


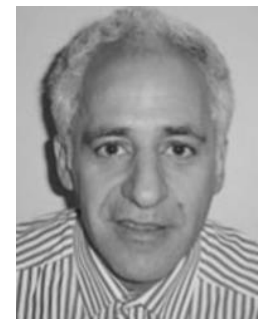

Jean Gaubert received the M.S. and the $\mathrm{Ph} . \mathrm{D}$. degrees in applied physics from Paul Sabatier University, Toulouse, France, in 1985, and 1988, respectively. From 1989 to 2001 he was an Assistant Professor at the Ecole Nationale Supérieure de l'Electronique et de ses Applications, Cergy-Pontoise, France, where he was involved with high-speed GaAs and InP bipolar devices modelling. In 2001, he joined Aix-Marseille University (AMU), France, and the Institut Matériaux Microélectronique Nanosciences de Provence (IM2NP UMR CNRS 7334) where is has been a full Professor since 2009. His research focuses on the design and integration of $\mathrm{RF} /$ Microwave CMOS integrated-circuits and systems for wireless communications.

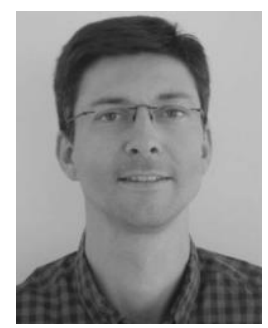

Nicolas Dehaese received the M.S degree from the ISEN engineering school, Lille, France, in 2002 and the Ph.D degree in electronics from the University of Provence, Aix-Marseille I, France, in 2005. Since 2006, he is an assistant professor in the Integrated Circuits Design Team from the Provence Nanosciences Microelectronics and Materials Laboratory (IM2NP), Marseille, France. His current field of research is in the design of analog/RF CMOS integrated circuits and system level specifications for low-cost applications including narrow band $(2.4 \mathrm{GHz})$ and Ultra Wideband (3.1-10.6 GHz) systems.

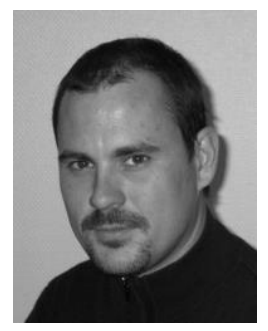

Stephane Meillere has received the Engineer degree in Microelectronics from the ISEN-Toulon, Institut Supérieur d'Electronique et du Numérique, School at Toulon in 2000 and the M.Sc. and Ph.D. degrees from the University of Provence Aix-Marseille I, France, in 2000 and 2004, respectively in Electronics and Electrical Engineering. He had worked as a Research Engineer at the ISEN-Toulon. Since 2005, he joined the University of Provence as an Assistant Professor. His research interests are mainly in the design of full custom ASICs. He integrated in the same time the Integrated Circuits Design Team at the IM2NP laboratory. His mean field of research is concentrated on analogue integrated circuits for radio frequency applications. His speciality is concentrated on base band and time domain circuits such as amplifiers, filters, oscillators, etc. The frequency range of applications goes from DC to Ultra-WideBand $(10 \mathrm{GHz})$. He participates in many revues and international conferences as a reviewer and in scientific committee. He worked on different research applications with industrial partners and ANR projects.

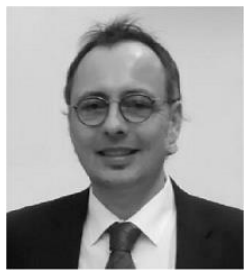

Herve Barthelemy has received the M.S. degree in Electrical Engineering in 1992 and the $\mathrm{PhD}$ degree in Electronics from the University of Paris XI Orsay, France in 1996. From 1996 to 2000 he was an Assistant Professor at the Institut Supérieur d'Electronique et du Numérique (ISEN'Toulon), France. In 2000 he joined the University of Provence where is has been a full Professor in 2005. Since September 2007, Prof. H. Barthélemy joined the University of Sud-Toulon-Var, France. He has served as Track Chair for the IEEE NEWCAS, MIDWEST, ICECS and ISCAS conferences. He was also the Technical Program co-Chair for the IEEE International conference IEEE NEWCAS 2011 and the General Chair of the IEEE ICECS 2014 Conference. Hervé Barthélemy serves as an associate editor of the journal IEEE Transactions Circuits \& Systems II during the period 20112012 and 2012-2013. He research interests include CMOS analog signal processing, radiofrequency, instrumentation and wireless sensors.

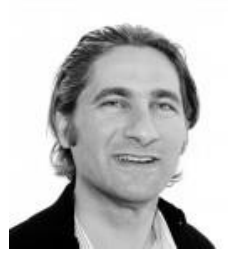

Laurent Ouvry has received the M.Eng. degree from Supelec in 1994 and the M.S. degree in RF, antennas, and digital signal processing and communications from Rennes I University in 1995 . He joined CEA-Leti in 1997 and is now is project leader on UWB wireless systems at CEALeti-Minatec. He led the Digital Communication Lab at CEA until 2010 and joined the RF design Lab afterwards. Since 2004, he has been responsible for impulse radio UWB low data rate projects at CEA-Leti and initiated system activities on ultra-low power for IEEE 802.15.4 compliant radios. He was involved in the key ICT IP projects on UWB and has been an active contributor to the IEEE802.15.4a UWB standard definition. He was also involved in ICT IP projects related to wireless sensor networks. Since 2007, he is working in the area of body area networks, was involved in the IEE802.15.6 standardization for BAN and coordinated a cooperative research project on the same matter. 